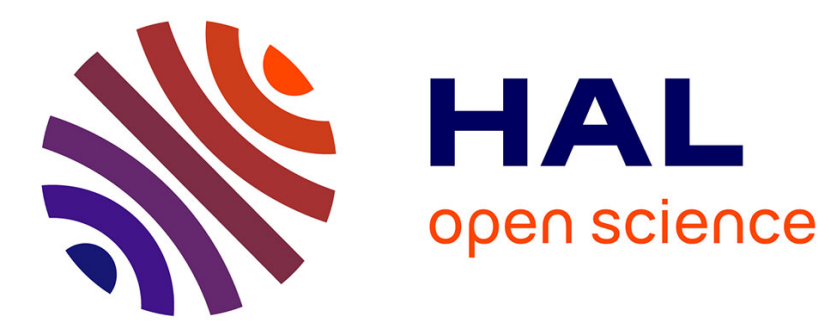

\title{
Neutron-rich In and Cd isotopes close to the doubly magic ${ }^{132} \mathrm{Sn}$
}

\author{
A. Scherillo, J. Genevey, J.A. Pinston, A. Covello, H. Faust, A. Gargano, R. \\ Orlandi, G.S. Simpson, I. Tsekhanovich, N. Warr
}

\section{- To cite this version:}

A. Scherillo, J. Genevey, J.A. Pinston, A. Covello, H. Faust, et al.. Neutron-rich In and Cd isotopes close to the doubly magic ${ }^{132}$ Sn. Physical Review C, 2004, 70, 054318 (10 p.). 10.1103/PhysRevC.70.054318 . in2p3-00023351

\section{HAL Id: in2p3-00023351 https://hal.in2p3.fr/in2p3-00023351}

Submitted on 1 Dec 2004

HAL is a multi-disciplinary open access archive for the deposit and dissemination of scientific research documents, whether they are published or not. The documents may come from teaching and research institutions in France or abroad, or from public or private research centers.
L'archive ouverte pluridisciplinaire HAL, est destinée au dépôt et à la diffusion de documents scientifiques de niveau recherche, publiés ou non, émanant des établissements d'enseignement et de recherche français ou étrangers, des laboratoires publics ou privés. 


\title{
Neutron-rich In and Cd isotopes close to the doubly-magic ${ }^{132} \mathrm{Sn}$
}

\author{
A. Scherillo, ${ }^{1,2}$ J. Genevey, ${ }^{3}$ J. A. Pinston, ${ }^{3, *}$ A. Covello,${ }^{4}$ H. Faust, ${ }^{1}$ A. \\ Gargano, ${ }^{4}$ R. Orlandi, ${ }^{1,5}$ G. S. Simpson, ${ }^{1}$ I. Tsekhanovich, ${ }^{1}$ and N. Warr ${ }^{2}$ \\ ${ }^{1}$ Institut Laue-Langevin, B.P. 156, F-38042 Grenoble Cedex 9, France \\ ${ }^{2}$ Institut für Kernphysik, Universität zu Köln, \\ Zülpicherstr. 77, D-50937 Köln, Germany. \\ 3 Laboratoire de Physique Subatomique et de Cosmologie, \\ IN2P3-CNRS/Université Joseph Fourier, F-38026 Grenoble Cedex, France \\ ${ }^{4}$ Dipartimento di Scienze Fisiche, Università degli studi di Napoli Federico II and INFN, \\ Complesso Universitario di Monte Sant'Angelo, via Cintia, I-80126 Napoli, Italy \\ ${ }^{5}$ Department of Physics and Astronomy, The University of Manchester, \\ Brunswick Street, M13 9PL, United Kingdom
}

(Dated: July 29, 2004)

\begin{abstract}
Microsecond isomers in the $\mathrm{In}$ and $\mathrm{Cd}$ isotopes, in the mass range $A=123$ to 130 , were investigated at the ILL reactor, Grenoble, using the LOHENGRIN mass spectrometer, through thermalneutron induced fission reactions of $\mathrm{Pu}$ targets. The level schemes of the odd-mass ${ }^{123-129} \mathrm{In}$ and new measurements of the $\mu$ s half-lives of the odd-odd ${ }^{126-130}$ In are reported. However, the expected $8^{+}$isomers in the even-mass $\mathrm{Cd}$ isotopes were not observed. The comparisons between the experimental $B(M 2)$ strengths for In and Sn isotopes are discussed. A shell-model study of the heaviest In and $\mathrm{Cd}$ nuclei was performed using a realistic interaction derived from the CD-Bonn nucleon-nucleon potential. Comparison shows that the calculated levels of ${ }^{130} \mathrm{In}$ and ${ }^{129} \mathrm{In}$ are in good agreement with the experimental values while some discrepancies occur for the lighter In isotopes. The collectivity of ${ }^{126,128} \mathrm{Cd}$ is discussed in the framework of the shell model and in comparison with ${ }^{204} \mathrm{Hg}$.

PACS numbers: 21.10.Tg, 23.20.Lv, 25.85.Ec, 27.60.+j
\end{abstract}

*Electronic address: pinston@lpsc.in2p3.fr 


\section{INTRODUCTION}

Experimental progress is currently being made in the region around doubly-magic ${ }^{132} \mathrm{Sn}$. However, nuclear structure information is more complete for nuclei above the $Z=50$ shellclosure [1] (for instance, $\mathrm{Sb}$ and Te isotopes) than for the In and $\mathrm{Cd}$ isotopes, which are much more difficult to produce. Only recently, Kautzsch et al. [2] have obtained some spectroscopic information on ${ }^{126,128} \mathrm{Cd}$, which have two proton and two and four neutron holes, respectively, inside the ${ }^{132} \mathrm{Sn}$ core. Although this information is still rather scarce, it seems to indicate that these nuclei possess some degree of collectivity. The authors of [2] take this as plausible evidence for a weakening of the shell structure. This is one of the reasons that spurred us to carry out a study of the neutron-rich nuclei of this region.

In the present work we searched for and studied the decay of $\mu$ s isomers in the neutronrich mass $A=123$ to 130 nuclei with the LOHENGRIN spectrometer at the ILL reactor in Grenoble. The aim was to complete the previous data on the heavy $\mathrm{Cd}$ and $\mathrm{In}$ isotopes. Apart from the above quoted study on the Cd isotopes, the low-spin levels up to $13 / 2$ in ${ }^{123-127}$ In were previously investigated from the $\beta$-decay of $\mathrm{Cd}$ isotopes [3, 4] and, very recently, high-spin ms isomers in ${ }^{125-129}$ In were discovered $[5,6]$. Preliminary reports were also presented by M. Hellström et al. $[7,8]$ on the search for $\mu$ s isomers in the heavy $\mathrm{Cd}$ and In isotopes at the FRS spectrometer at GSI, but no level schemes were proposed.

Motivated by the new data from the present experiment, we have performed calculations to test the ability of the shell model to describe the heavy $\mathrm{Cd}$ and $\mathrm{In}$ isotopes, with proton and neutron holes outside the ${ }^{132} \mathrm{Sn}$ core. In this work, a realistic effective interaction derived from the CD-Bonn nucleon-nucleon potential [9] is used. Similar calculations were performed in Ref. [10] for nuclei with proton particles and neutron holes around ${ }^{132} \mathrm{Sn}$, and in Ref. [11] for ${ }^{129} \mathrm{In}$. In both cases good agreement with the experimental data was found.

The paper is organized as follows. In Sec. II we describe the experimental procedure, while in Sec. III the results of our measurements are presented. In Sec. IV the level schemes of odd In isotopes, as they result from different experimental studies, are reported and discussed. Sec. $\mathrm{V}$ is devoted to the comparison of the results of our shell-model calculations with the experimental data. In Sec. VI all the available information on $M 2$ and E3 transitions in heavy In and Sn isotopes is summarized. Section VII contains a summary of our conclusions. 


\section{EXPERIMENTAL PROCEDURE}

Two different experiments have been performed to explore the In and Cd region in the vicinity of ${ }^{132} \mathrm{Sn}$. In the first one, devoted to the odd masses, both the conversion electrons and $\gamma$-rays were measured while in the second, devoted to the even masses, only $\gamma$-rays were measured.

The nuclei of mass $A=123,125,127$ were produced by thermal-neutron induced fission of ${ }^{239} \mathrm{Pu}$ and ${ }^{241} \mathrm{Pu}$. The LOHENGRIN mass spectrometer has been used to separate the fission fragments (FFs) recoiling from a thin target of about $400 \mu \mathrm{g} / \mathrm{cm}^{2}$, according to their mass to ionic charge ratios $(A / q)$. The FFs were detected in a gas detector of $13 \mathrm{~cm}$ length, and subsequently stopped in a $12 \mu \mathrm{m}$ thick Mylar foil. Behind the foil, two cooled adjacent $\mathrm{Si}(\mathrm{Li})$ detectors covering an area of $2 \times 6 \mathrm{~cm}^{2}$ were placed to detect the conversion electrons and X-rays de-exciting the isomers, while the $\gamma$-rays were detected by two large-volume Ge detectors placed perpendicular to the beam. Details on the experimental set-up can be found in $[1,12]$.

To produce the nuclei of mass $A=126,128,130$ a thin target of ${ }^{241} \mathrm{Pu}$ of about $400 \mu \mathrm{g} / \mathrm{cm}^{2}$ was used. The FFs were detected in a $\Delta E-E$ gas detector to achieve very good mass resolution, and the $\gamma$-rays in two germanium detectors: one Clover detector (efficiency $150 \%$ ) and one triple cryostat of the Miniball array [13]. The efficiency for $\gamma$-ray detection of the second set-up was higher than the first one, making the study of nuclei with low production rates possible.

\section{EXPERIMENTAL RESULTS}

\section{A. ${ }^{123} \mathbf{I n}$}

A new $\mu$ s isomer has been found in ${ }^{123} \mathrm{In}$. In the $\gamma$-decay spectrum of the mass $A=123$ in delayed coincidence with the FFs (see fig. 1), four new $\gamma$-lines (880.7, 1019.0, 1027.6 and $1166.0 \mathrm{keV}$ ), not belonging to the already known $6 \mu$ s isomer of ${ }^{123} \mathrm{Sn}[14]$ are present. Two $\gamma$-rays of 1027.5 and $1165.9 \mathrm{keV}$ respectively, were previously observed in $\beta$-decay studies of ${ }^{123} \mathrm{Cd}$ to ${ }^{123} \mathrm{In}$ [4]. They de-excite two levels of ${ }^{123} \mathrm{In}$ with possible spin and parity assignments of $11 / 2^{+}$and $13 / 2^{+}$respectively. The fact that we observe two transitions with almost the same energy as those in ${ }^{123} \mathrm{In}$, and the absence of any common $\gamma$-rays belonging to ${ }^{123} \mathrm{Sn}$ 

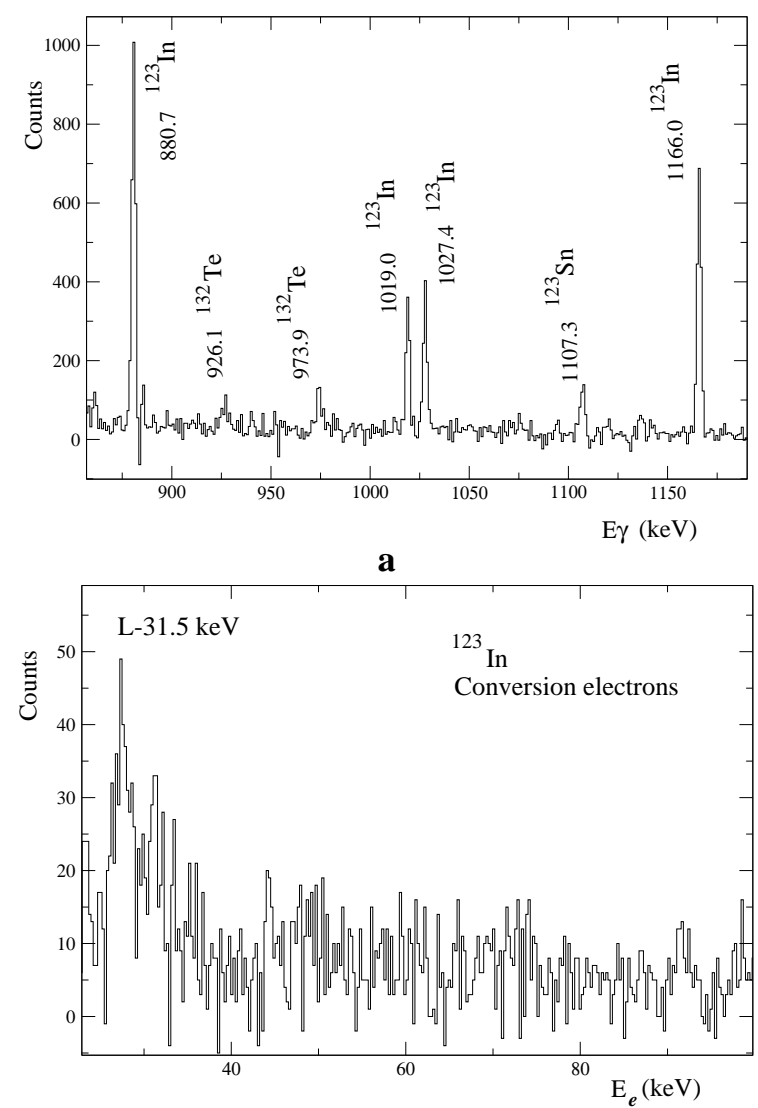

b

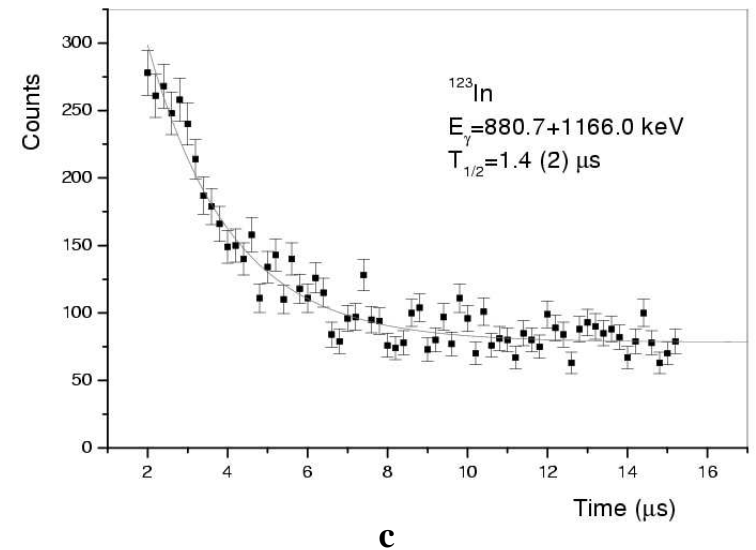

FIG. 1: a) $\gamma$-decay spectrum of the mass $A=123$ in delayed coincidence with the fission fragments. b) $\mathrm{Si}(\mathrm{Li})$ spectrum of the mass $A=123$ in delayed coincidence with the fission fragments. c) Time spectrum of 880.7 and $1166.0 \mathrm{keV}$.

allow this isomer to be attributed to ${ }^{123} \mathrm{In}$, the only element along with ${ }^{123} \mathrm{Sn}$ which has sufficient production yield. The $\gamma$-ray of $\sim 138 \mathrm{keV}$ connecting the $13 / 2^{+}$level to the $11 / 2^{+}$ level was not observed, due to the low expected relative intensity $(\sim 6)$ deduced from the intensities of the observed transitions reported in table I. 


\begin{tabular}{ccc}
\hline \hline Transition & Transition's energy & Intensity \\
\hline$\left(13 / 2^{-}\right) \rightarrow 13 / 2^{+}$ & 880.7 & 107 \\
$\left(13 / 2^{-}\right) \rightarrow 11 / 2^{+}$ & 1019.0 & 40 \\
$11 / 2^{+} \rightarrow 9 / 2^{+}$ & 1027.4 & 42 \\
$13 / 2^{+} \rightarrow 9 / 2^{+}$ & 1166.0 & 100 \\
\hline \hline
\end{tabular}

The half-life of the isomer is $1.4(2) \mu \mathrm{s}$, as shown in fig. 1. Due to the poor statistics, it was not possible to observe the conversion electrons in coincidence with the $\gamma$-rays of the isomeric decay. However, in the $\mathrm{Si}(\mathrm{Li})$ spectrum in delayed coincidence with the FFs of mass $A=123$, an electron line of $27.5 \mathrm{keV}$ decaying with a half-life comparable to the $\gamma$ cascaded was observed (see fig. 1). The non-observation of another electron group suggests that this line corresponds to L conversion electrons of a transition of $31.5 \mathrm{keV}$. A value so close to the binding energy of the $\mathrm{K}$ electrons explains the non-observation of the K-X rays. Assuming that this low-energy transition has an $E 2$ multipolarity, a value of $B(E 2)=3.3(5)$ W.u. can be deduced.

\section{B. ${ }^{127}$ In}

We have observed in the present work a 9.0(6) $\mu$ s isomer which decays by a cascade consisting of a strongly-converted $E 2$ transition of $47 \mathrm{keV}$, and two $\gamma$-rays of 221 and $233 \mathrm{keV}$ in coincidence one with the other (fig. 2). The two $\gamma$-rays were the same as those first observed by Hellström et al. [7], and the reported value of the half-life $13(2) \mu \mathrm{s}$ is in rough agreement with that found in this work (see table II). Moreover, these authors also observed an abnormally high number of counts in the first $500 \mathrm{~ns}$ interval of the time spectrum and suggested the possible presence of two isomeric states in ${ }^{127} \mathrm{In}$. We have not observed the short component in our data and we feel that, if it exists, its half-life is shorter than $\sim 0.5 \mu$ s. The $\mathrm{Si}(\mathrm{Li})$ spectrum obtained in coincidence with any of these two lines (fig. 2) shows the characteristic indium X-rays, and the $\mathrm{K}$ and L conversion electrons of the isomeric transition. The multipolarity of the transition is $E 2$, and $B(E 2)=0.3$ W.u. was deduced. 

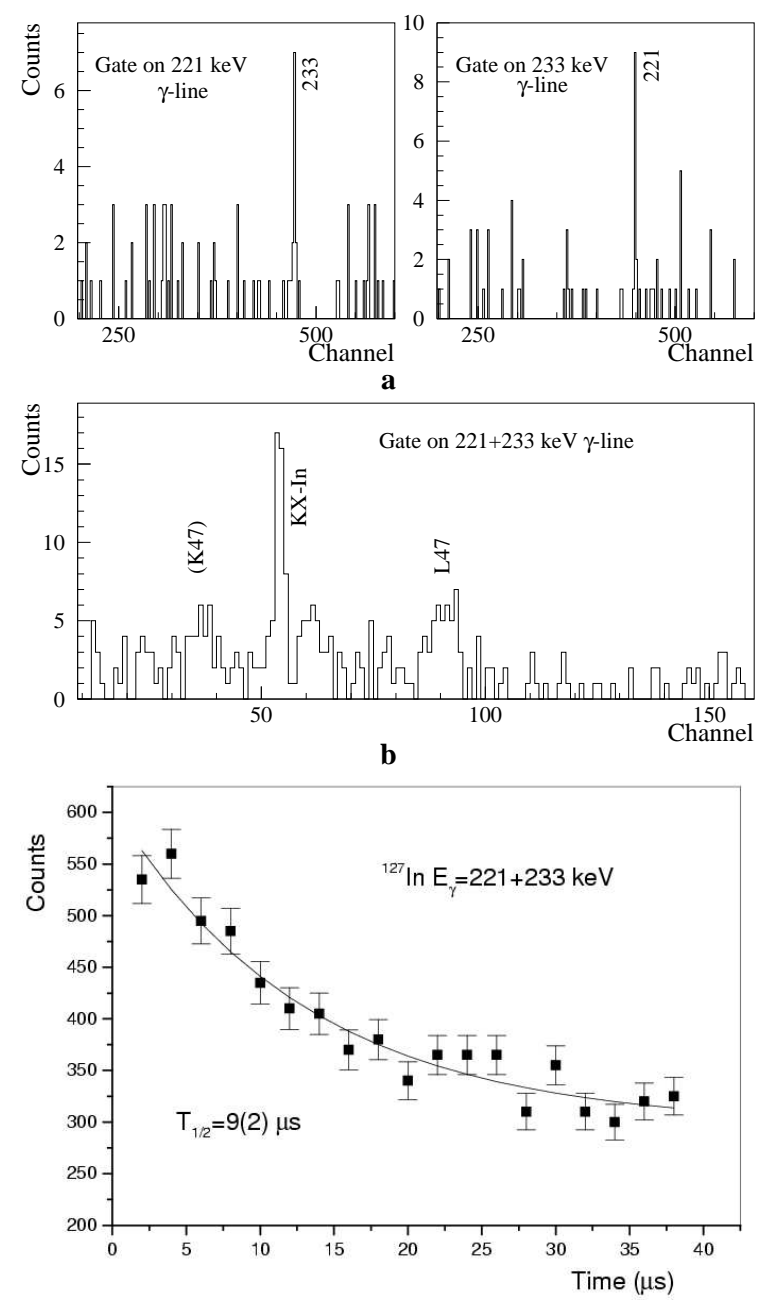

c

FIG. 2: a) Coincidence spectra gated on the $\gamma$-ray of 233 or $221 \mathrm{keV}$. b) $\mathrm{Si}(\mathrm{Li})$ spectrum obtained in coincidence with the $\gamma$-rays of 221 and $233 \mathrm{keV}$. c) Time spectrum of 221 and $233 \mathrm{keV}$.

\section{Odd-odd Indium isotopes}

In this work, the half-lives of the previously-known isomeric transitions in ${ }^{130} \mathrm{In}[7],{ }^{128} \mathrm{In}$ [15] and ${ }^{126}$ In [7] were measured. The result of this work is reported in table II, where the values obtained in previous works $[7,8]$ are also shown. In the case of ${ }^{126} \mathrm{In}$, our value is

shorter than the previous measurement, whereas in the case of ${ }^{130} \mathrm{In}$ and ${ }^{128} \mathrm{In}$ our values are much more precise. 
TABLE II: Half-lives measured in the present work along with the previous data.

\begin{tabular}{cccc}
\hline \hline Nucleus & Transition & $\begin{array}{c}\text { half-life }(\mu \mathrm{s}) \\
\text { This Work }\end{array}$ & $\begin{array}{c}\text { half-life }(\mu \mathrm{s})^{a} \\
\text { Previous Value }\end{array}$ \\
\hline${ }^{123} \mathrm{In}$ & $17 / 2^{-} \rightarrow 13 / 2^{-}$ & $1.4(2)$ & $<0.5$ and $13(1)$ \\
${ }^{127} \mathrm{In}$ & $29 / 2^{+} \rightarrow 25 / 2^{+}$ & $9.0(6)$ & $29(2)$ \\
${ }^{126} \mathrm{In}$ & $1^{-} \rightarrow 3^{+}$ & $22(2)$ & $175(90)$ \\
${ }^{128} \mathrm{In}$ & $1^{-} \rightarrow 3^{+}$ & $23(2)$ & $1-10$ \\
${ }^{130} \mathrm{In}$ & $3^{+} \rightarrow 1^{-}$ & $3.1(3)$ & \\
\hline \hline
\end{tabular}

${ }^{a}$ see $[7]$

\section{Cadmium isotopes}

Two $\mu$ s isomer were reported by Hellström et al. [8] in the odd-mass Cd isotopes, one in ${ }^{127} \mathrm{Cd}$ and the other in ${ }^{125} \mathrm{Cd}$. We have also seen the two $\gamma$ lines of 720 and $743 \mathrm{keV}$ belonging to ${ }^{125} \mathrm{Cd}$, but not the $\gamma$-ray of $830 \mathrm{keV}$ in ${ }^{127} \mathrm{Cd}$.

Furthermore, as the GSI group, we have not observed the $\mu$ s isomers in the even-mass $\mathrm{Cd}$ isotopes. If ${ }^{130} \mathrm{Cd}$, with the predicted fission yield lower than $10^{-6}$, is not experimentally observable, ${ }^{126,128} \mathrm{Cd}$, with fission yields $\sim 10^{-4}$, comparable to the yield of the observed ${ }^{125} \mathrm{Cd}$, should be observed. Consequently, we can exclude the presence of $\mu$ s isomers with half-lives longer than $0.5 \mu \mathrm{s}$ in the heavy $\mathrm{Cd}$ isotopes.

\section{LEVEL SCHEMES OF ODD-IN NUCLEI}

The heavy In and Cd nuclei, with neutron and proton holes inside the ${ }^{132} \mathrm{Sn}$ core, are characterized by the presence of two high-spin states, $\pi g_{9 / 2}^{-1}$ and $\nu h_{11 / 2}^{-1}$, at low excitation energy. The $p-n$ interaction in the $\left(\pi g_{9 / 2}^{-1} \nu h_{11 / 2}^{-1}\right)_{10^{-}}$state is very strong and is expected to produce very perturbed yrast lines. These features greatly complicate the construction of the level schemes and therefore different experimental techniques are needed to study these nuclei. The level schemes of ${ }^{123-129}$ In shown in fig. 4 are the result of the synthesis of different works: the ms isomer experiments performed at the OSIRIS mass separator [5, 6], and the $\mu$ s isomer experiments performed with the FRS at GSI $[7,8]$ and the LOHENGRIN 


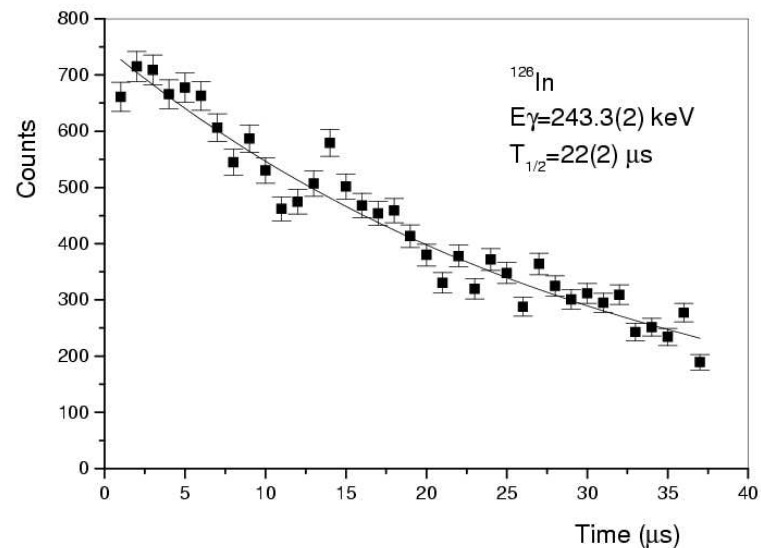

a

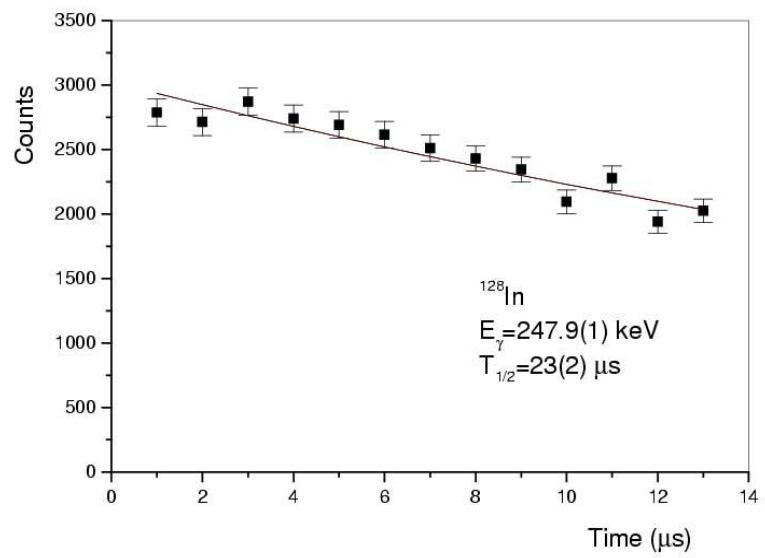

b

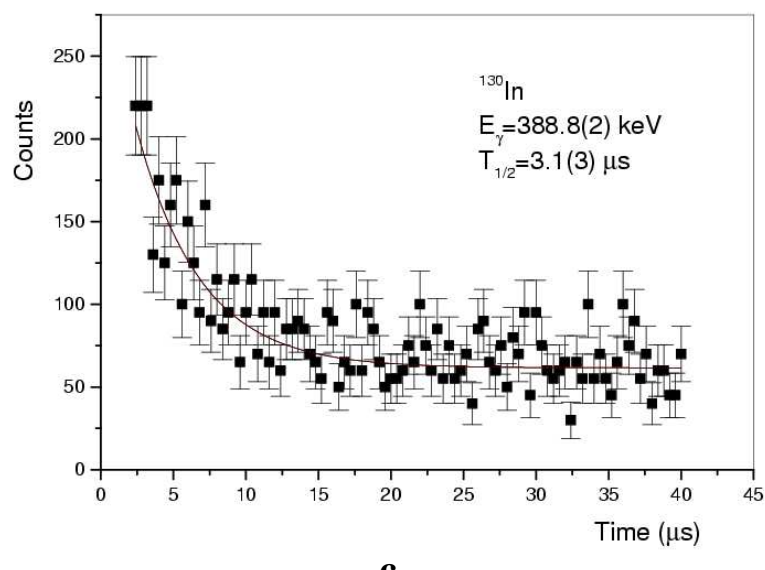

c

FIG. 3: Time spectrum of ${ }^{126,128,130} \mathrm{In}$.

spectrometer $[1,11]$. All the reported levels are in the vicinity of the yrast line, therefore the low-spin levels fed in previous works by $\beta$-decay experiments are not shown in fig. 4 . 


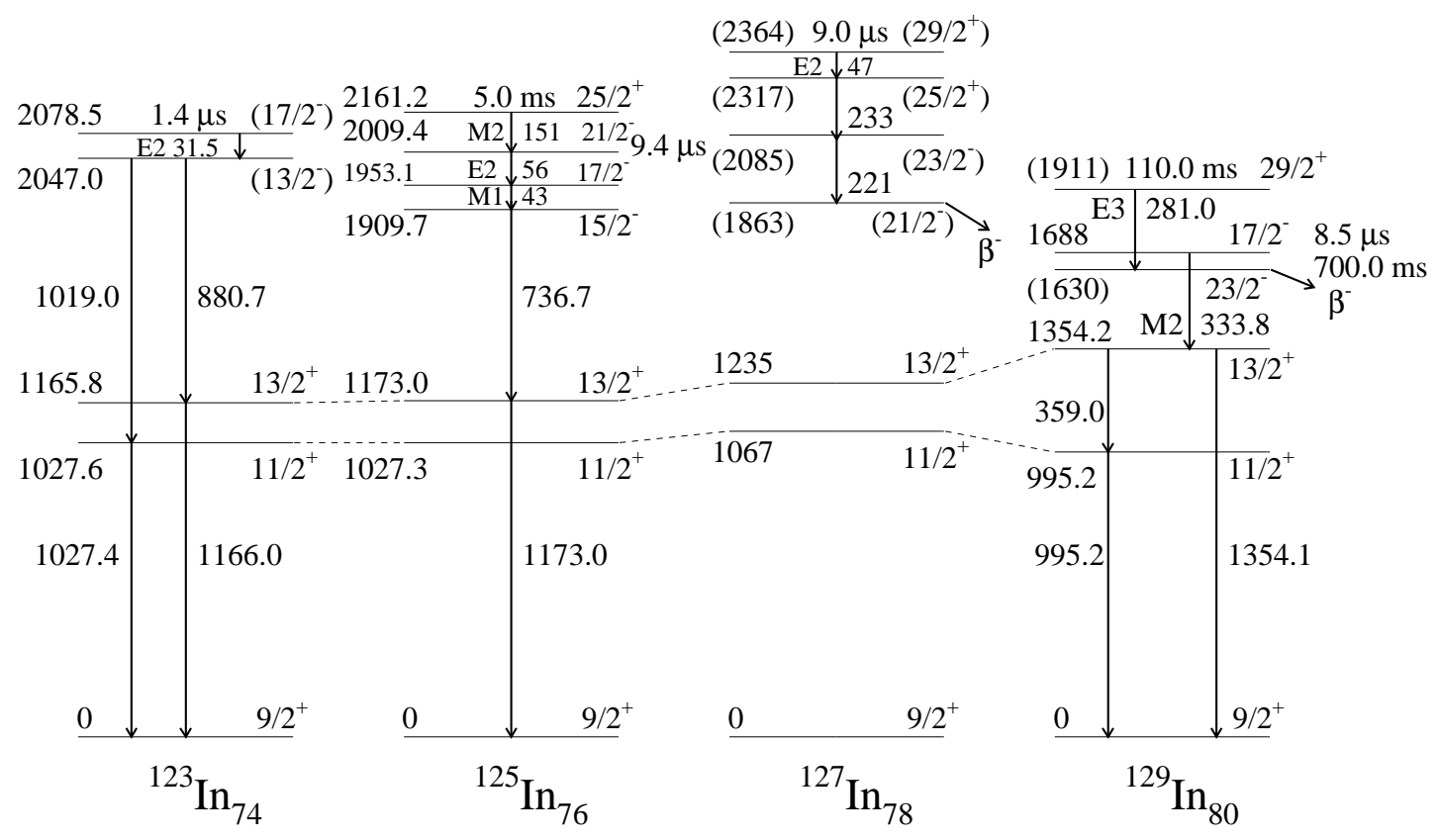

FIG. 4: Level schemes of ${ }^{123,125,127,129}$ In. The level energies in parenthesis are deduced from $\beta$ spectra [6] and have large uncertainties.

\section{A. ${ }^{129} \mathbf{I n}$}

In ${ }^{129} \mathrm{In}, \mathrm{ms}$ and $\mu \mathrm{s}$ isomers are present. Fogelberg et al. [5] have shown evidence of a high-spin $29 / 2^{+}$yrast trap which decays by an E3 transition (110 ms half-life) to another yrast trap of spin $23 / 2^{-}$(half-life $700 \mathrm{~ms}$ ), which $\beta$ decays to ${ }^{129} \mathrm{Sn}$. More recently a more precise value of the excitation energy, $1630(56) \mathrm{keV}$, was measured for the $23 / 2^{-}$state from the $\beta$-decay spectra [6].

Genevey et al. [11] have shown evidence of a $17 / 2^{-}$isomer of $8.5 \mu \mathrm{s}$, which decays by a $\gamma$ cascade to the $9 / 2^{+}$ground state.

\section{B. ${ }^{127}$ In}

The isomeric cascade observed in this isotope has no overlap with the previously known $\gamma$ rays which feed directly or indirectly the $1 / 2^{-}$isomer of $420(65) \mathrm{keV}$ energy $[3,6]$. Moreover, the comparison with the neighboring ${ }^{125} \mathrm{In}$ and ${ }^{129} \mathrm{In}$ shows that the excited states above the $9 / 2^{+}$ground state have energies higher than about $1 \mathrm{MeV}$, which excludes the possibility that the observed cascade ends at this level. Consequently, this cascade can only de-excite 
a $29 / 2^{+}$isomer to a $21 / 2^{-}$state, decaying itself by $\beta$ emission. This finding agrees with the measurement of Gausemel et al. [6] who have found a $21 / 2^{-}$isomer in ${ }^{127} \mathrm{In}$, of 1.0 s half-life and have deduced its energy, $1863(58) \mathrm{keV}$, from $\beta$-decay spectra.

Two other states at energies of $1067 \mathrm{keV}$ and $1235 \mathrm{keV}$, feeding directly the $9 / 2^{+}$ground state are added to the level scheme. They were reported by Hoff et al. [3] but without spin and parity assignments. By analogy with ${ }^{125} \mathrm{In}$ and ${ }^{129} \mathrm{In}$, spin and parity assignments of $11 / 2^{+}$and $13 / 2^{+}$, respectively, are proposed for these two states.

\section{C. ${ }^{125}$ In}

Fogelberg et al. [5] have found a $5 \mathrm{~ms}$ isomer in ${ }^{125} \mathrm{In}$ which decays by an $M 2$ transition to a $9.4 \mu$ s isomer which itself decays by a $\gamma$-ray cascade to the $9 / 2^{+}{ }^{125}$ In ground state. We have also observed the decay of the $\mu$ s isomer and agree with the previous level scheme and the E2 and $M 1$ mutipolarities measured for the 56 and $43 \mathrm{keV}$ transitions respectively. Fogelberg et al. have suggested a spin and parity assignment of $23 / 2^{-}$for the ms isomer, and positive parities for the states below the isomer. However, this hypothesis seems inconsistent with the non-observation of the cross over between the $1953.1 \mathrm{keV}$ and $1173.0 \mathrm{keV}$ levels and between the 1909.7 and $1027.3 \mathrm{keV}$ levels respectively. A 25/2 ${ }^{+}$spin and parity assignment for the ms isomer and a change of parity for the three successive levels seem necessary to explain the non-observation of the cross over in the experimental data.

\section{D. ${ }^{123} \mathbf{I n}$}

The isomer in ${ }^{123}$ In decays by an $E 2$ transition and a $\gamma$-ray cascade to the $9 / 2^{+}$ground state. The simultaneous feeding of the $11 / 2^{+}$and $13 / 2^{+}$levels at 1027.4 and $1165.8 \mathrm{keV}$, respectively, suggests a spin and parity assignment $13 / 2^{-}$or $15 / 2^{+}$for the $2047.5 \mathrm{keV}$ level. The negative parity assignment is preferred by analogy with the heavier In isotopes and is reported in fig. 4 , but a positive parity cannot be completely ruled out. 


\section{SHELL MODEL CALCULATIONS IN INDIUM AND CADMIUM ISOTOPES}

Our shell model study of the indium and cadmium isotopes has been performed using a realistic effective interaction derived from the CD-Bonn nucleon-nucleon potential. The ${ }^{132} \mathrm{Sn}$ nucleus was considered as a closed core, the proton and neutron holes occupying the levels in the 28-50 shell and the 50-82 shell, respectively. We have assumed an effective proton charge $\mathrm{e}_{\pi}=1.35 e$, as a result of a study performed on the $\mathrm{N}=50$ isotones [16], and an effective neutron charge $\mathrm{e}_{\nu}=0.78 e$, which reproduces the $B\left(E 2 ; 10^{+} \rightarrow 8^{+}\right)$in ${ }^{130} \mathrm{Sn}$. Details on the calculation can be found in [11] and references therein. The results presented in this section have all been obtained by using the OXBASH shell-model code [17].

\section{A. Odd In nuclei}

In fig. 5a, the experimental and calculated levels of ${ }^{129} \mathrm{In}$ and ${ }^{130} \mathrm{Sn}$ are compared. The results for ${ }^{129}$ In have been previously reported in [11]. For ${ }^{129} \mathrm{In}$, all the experimental levels, except the $1 / 2^{-}$at $369 \mathrm{keV}$, are shown, while in the spectrum of ${ }^{130} \mathrm{Sn}$ only some selected yrast levels are included. The dominant configurations of all these states are also indicated. It should be mentioned, however, that a significant configuration mixing is present in some states. More precisely, only the $7^{-}$and $10^{+}$state in ${ }^{130} \mathrm{Sn}$ and the $11 / 2^{-}, 23 / 2^{-}, 17 / 2^{-}$, and $29 / 2^{+}$states in ${ }^{129}$ In have a weight of the dominant configuration larger than about $85 \%$. The excitation energies in ${ }^{130} \mathrm{Sn}$ are rather well reproduced by the shell-model calculations. However, it is interesting to note that the first $2^{+}$state is overestimated by $162 \mathrm{keV}$. This is a common feature in this region, and it may be traced to the model-space truncation.

The experimental levels of ${ }^{129} \mathrm{In}$ are expected to result from the coupling of a $\pi g_{9 / 2}$ hole to the two-neutron hole states in ${ }^{130} \mathrm{Sn}$. The observed decrease in energy of the $29 / 2^{+}$aligned state with respect to the $10^{+}$in ${ }^{130} \mathrm{Sn}$ is explained by the strong $p-n$ interaction in the $\left(\nu h_{11 / 2}^{-1} \pi g_{9 / 2}^{-1}\right)_{10^{-}}$state. An analogous effect is observed for the other aligned state, $J^{\pi}=$ $23 / 2^{-}$, of dominant configuration $\pi g_{9 / 2}^{-1} \nu h_{11 / 2}^{-1} \nu d_{3 / 2}^{-1}$. However, this effect is weaker because the $p-n$ interaction in the $\left(\pi g_{9 / 2}^{-1} \nu d_{3 / 2}^{-1}\right)_{6^{+}}$state is less attractive than in the $\left(\pi g_{9 / 2}^{-1} \nu h_{11 / 2}^{-1}\right)_{10^{-}}$ state. This strong decrease in energy of the $23 / 2^{-}$and $29 / 2^{+}$is responsible for these two states to be long-lived isomers. This is very well reproduced by the shell-model calculation which also predicts $\mu$ s isomerism for the $17 / 2^{-}$state, which decays by an $E 2$ transition. 


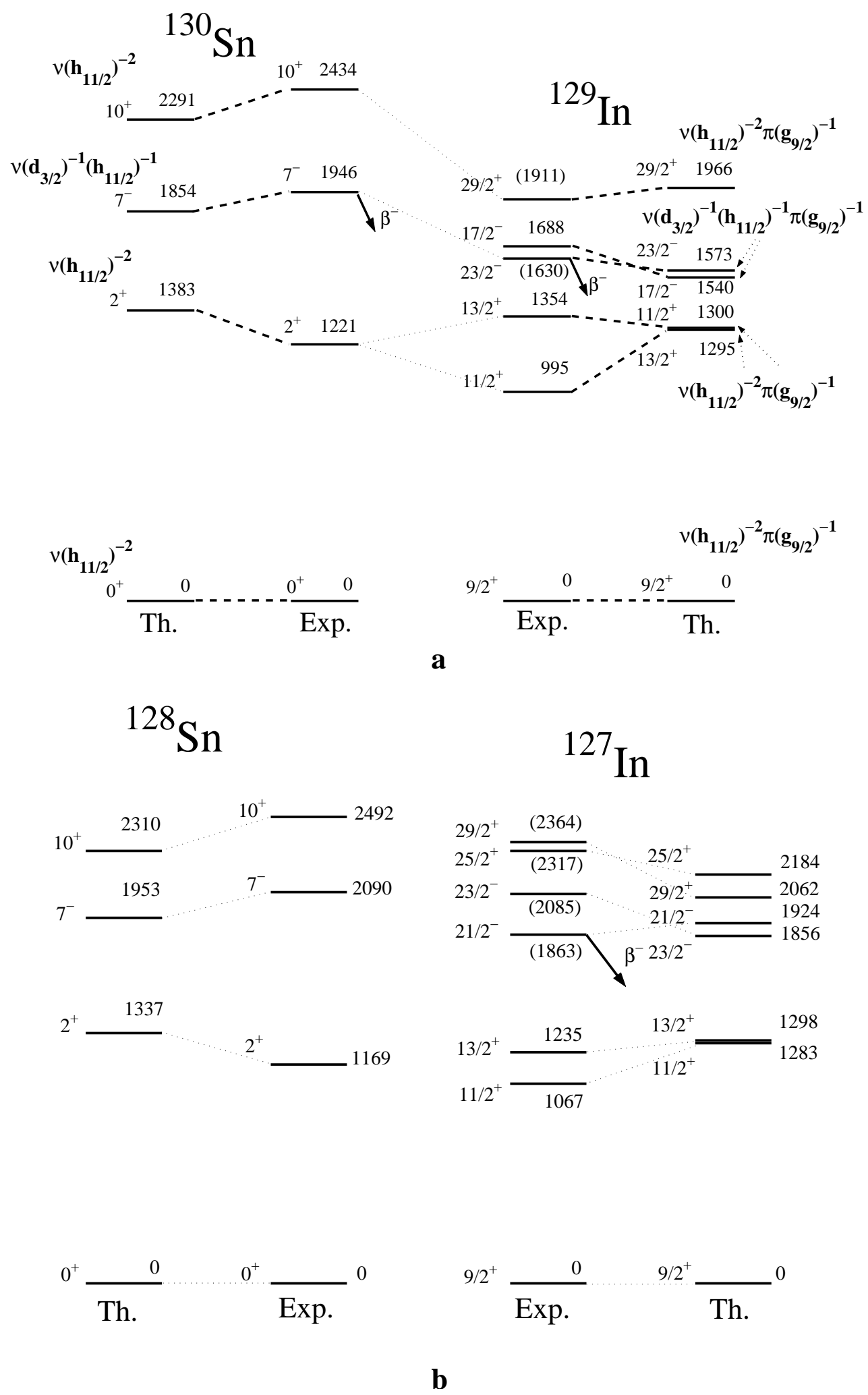

FIG. 5: Experimental and calculated energies for ${ }^{129} \mathrm{In}$ and ${ }^{130} \mathrm{Sn}$ (a), and for ${ }^{127} \mathrm{In}$ and ${ }^{128} \mathrm{Sn}$ (b). 
The main discrepancy between experiment and theory occurs for the $11 / 2^{+}$level, which is overestimated by $300 \mathrm{keV}$. This is not surprising since it originates essentially from the coupling of the $2^{+}$state in ${ }^{130} \mathrm{Sn}$ to the $g_{9 / 2}$ proton hole, the energy of the former, as we have already seen, being overestimated by the theory.

From fig. $5 \mathrm{~b}$ it appears that the observed $29 / 2^{+}$and $23 / 2^{-}$states in ${ }^{127}$ In are closer to the $10^{+}$and $7^{-}$in ${ }^{128} \mathrm{Sn}$, respectively, as compared to what is shown in fig. 5a. This

effect could be explained by a decrease in the effects of the $p-n$ interaction from ${ }^{129} \mathrm{In}$ to ${ }^{127} \mathrm{In}$. This is underestimated by the theory, indicating that the distribution of the two extra neutron holes may not be properly described. Another feature, possibly related to the effects of the $p-n$ interaction, is the inversion of the $29 / 2^{+}$and $25 / 2^{+}$, and $23 / 2^{-}$and $21 / 2^{-}$levels respectively, in the calculated spectrum of ${ }^{127} \mathrm{In}$. As a consequence, a $29 / 2^{+}$ ms isomer decaying by an $E 3$ transition is predicted, whilst a $\mu$ s one decaying by an $E 2$ transition is measured. Moreover, the theory predicts a $23 / 2^{-} \beta$-decaying isomer, whilst a $21 / 2^{-}$one is measured [6]. It should be mentioned that in the energy interval 1.8-2.4 MeV the theory predicts several high-spin yrast states. However, we have not reported them in fig. 5b since the spin-parity assignments based on the experimental findings (see Sec. IIIB) are to be considered firm. While it would be very interesting to compare experiment and theory for ${ }^{125} \mathrm{In}$ and ${ }^{123} \mathrm{In}$, the number of configurations is too large to allow calculations with the OXBASH code.

\section{B. Even In nuclei}

Nuclear structure information is very scarce for the heavy odd-odd In nuclei. However, the $1^{-}, 3^{+}$and $1^{+}$states, resulting from the coupling of a proton hole $g_{9 / 2}$ with the neutron holes $h_{11 / 2}, d_{3 / 2}$ or $g_{7 / 2}$, respectively, are experimentally known $[18,19]$ in ${ }^{126-130}$ In. In these three states the neutron and proton are in coplanar orbits and the $p-n$ interaction is expected to become strongly attractive, in particular for the $1^{+}$state. A weaker interaction is expected for the $3^{+}$state where a $d_{3 / 2}$ neutron is involved, and this level is used to normalize the level schemes of fig. 6. The shell-model calculations reproduce rather well the relative energies of the three levels in ${ }^{130} \mathrm{In}$ as well as their evolution when going from ${ }^{130} \mathrm{In}$ to ${ }^{126} \mathrm{In}$. It is interesting to note the strong variation of the position of the $1^{-}$state from ${ }^{130} \mathrm{In}$ to ${ }^{128} \mathrm{In}$. This variation could be explained by a decrease in the effects of $p-n$ interaction when 


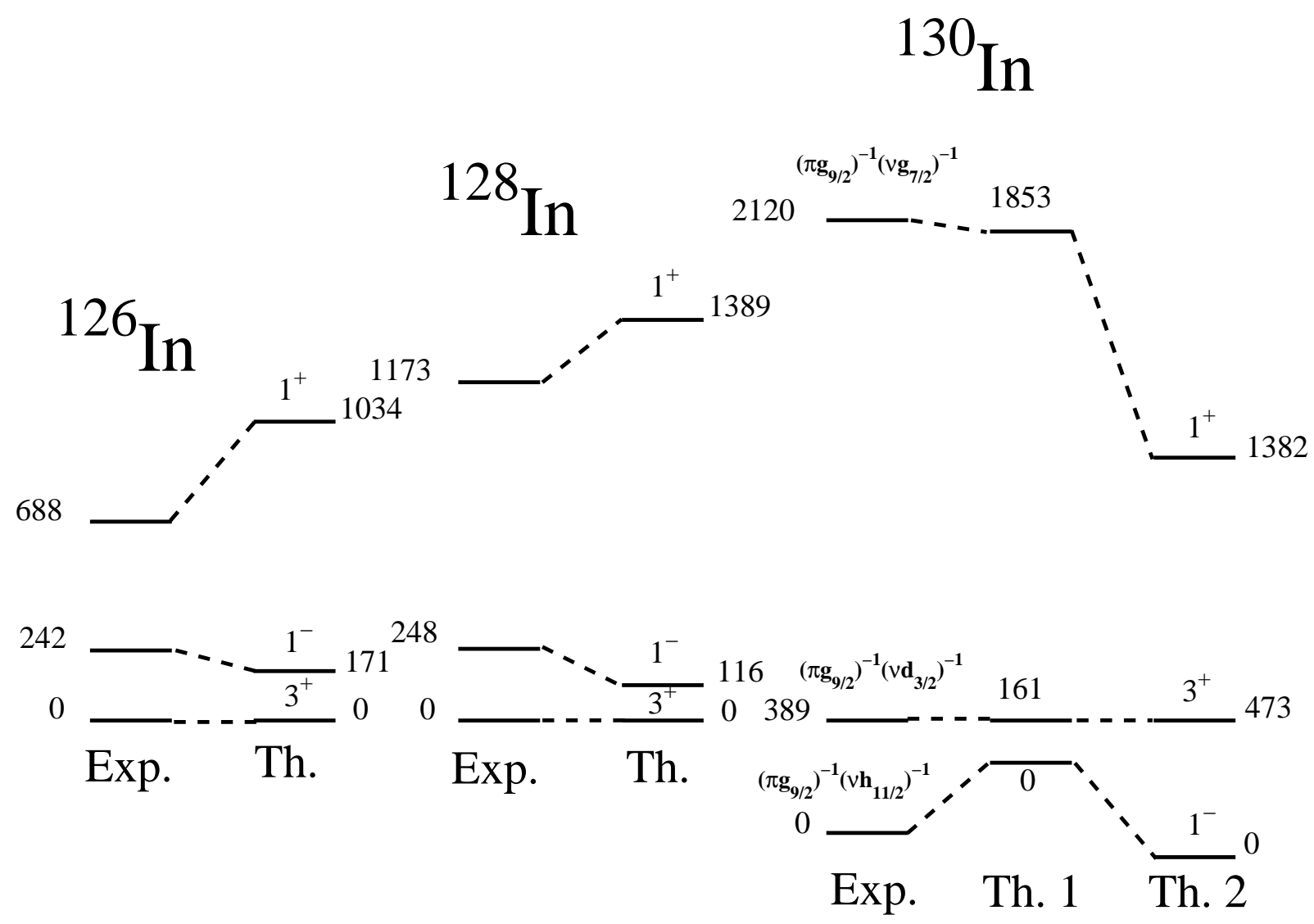

FIG. 6: Experimental and calculated energies for the odd-odd In.

increasing the number of neutron holes. However, as for the aligned $29 / 2^{+}$state in ${ }^{127} \mathrm{In}$, the decrease is underestimated by the calculation.

The very recent results of a shell-model calculation for ${ }^{130} \mathrm{In}$ by Dillmann et al. [18] are also reported in fig. 6 (Th2). Although in this work the two-body matrix elements are also derived from the CD-Bonn potential, the energy of the $1^{+}$state is underestimated by $738 \mathrm{keV}$, which is in strong disagreement with the outcome of our calculation.

In conclusion, the shell model reproduces rather well the levels of ${ }^{129} \mathrm{In}$ and ${ }^{130} \mathrm{In}$. However, it underestimates the decrease in the effects of the $p-n$ interaction when two neutrons are removed from ${ }^{130} \mathrm{In}$ or ${ }^{129} \mathrm{In}$. These results show the limits of the predictive power of the present shell-model calculations in the vicinity of ${ }^{132} \mathrm{Sn}$ for $N<82$. 


\section{Even Cd isotopes}

In the vicinity of the two closed shells of ${ }^{132} \mathrm{Sn}$ the $\mu$ s isomers are very abundant and disappear rapidly far from them [1]. However, below $Z=50$ they disappear suddenly for the $\mathrm{Cd}$ isotopes, no isomers having been identified up to now in the even-mass ones.

Neutron-rich ${ }^{126} \mathrm{Cd}$ and ${ }^{128} \mathrm{Cd}$ isotopes were recently produced [2] at ISOLDE from the beta-decay of $\mathrm{Ag}$ isotopes. However, only the first $2^{+}$and $4^{+}$states were identified and are shown in fig. 7 . The value of $E\left(4^{+}\right) / E\left(2^{+}\right) \sim 2.2$ suggests that some degree of collectivity is present in these two nuclei. However, the authors of [2] have taken this as a possible evidence for a weakening of the spherical $N=82$ neutron shell below ${ }^{132} \mathrm{Sn}$.

Our shell-model predictions for these two nuclei are reported in fig. 7. The comparison between experiment and theory shows that while the energies of both the $2^{+}$and $4^{+}$states are overestimated the $4^{+} \rightarrow 2^{+}$energy difference is correctly reproduced. The $E 2$ transition rates are also reported. Unfortunately, it is not possible to compare them with the experimental data, but the $B(E 2)$ values and the energy of the $8^{+} \rightarrow 6^{+}$transitions allow us to predict a half-life of about $10 \mathrm{~ns}$ for the $8^{+}$state in both $\mathrm{Cd}$ isotopes. This value is much shorter than the time of flight of $2 \mu \mathrm{s}$ of the FFs through the LOHENGRIN spectrometer and could explain the non-observation of these isomers in our work.

It is now interesting to compare ${ }^{128} \mathrm{Cd}$ with ${ }^{204} \mathrm{Hg}$ (fig 8), since the latter has two neutron and two proton holes inside doubly-magic ${ }^{208} \mathrm{~Pb}$ (fig 8) and possesses some degree of collectivity. For ${ }^{204} \mathrm{Hg}$, which is easier to study because it is on the line of stability, the energy levels and $E 2$ transition rates have been reported in the literature. This nucleus presents a nice collective band based on the ground state and is characterized by a $E\left(4^{+}\right) / E\left(2^{+}\right) \sim 2.6$ ratio, which is larger than the one measured in ${ }^{128} \mathrm{Cd}$. Rydström et al. [20] have shown that for ${ }^{204} \mathrm{Hg}$ the energy levels and the $E 2$ transition rates up to the $6^{+}$state are well reproduced by the shell model. These authors have also predicted that the collective band ends at the $8^{+}$state and that the states up to the $6^{+}$one are mostly built from the low-spin single-hole $s_{1 / 2}$ and $d_{3 / 2}$ orbits for the protons, and $p_{1 / 2}, f_{5 / 2}$ and $p_{3 / 2}$ orbits for the neutrons. Both these sets of single-parity states are close to the Fermi level, while the unique parity states have about $1.5 \mathrm{MeV}$ of excitation energy. The situation is different in ${ }^{128} \mathrm{Cd}$, because the unique-parity states are very close to the Fermi level. This feature may play an important role in the structure of this nucleus, and could perhaps explain its weaker collectivity. How- 

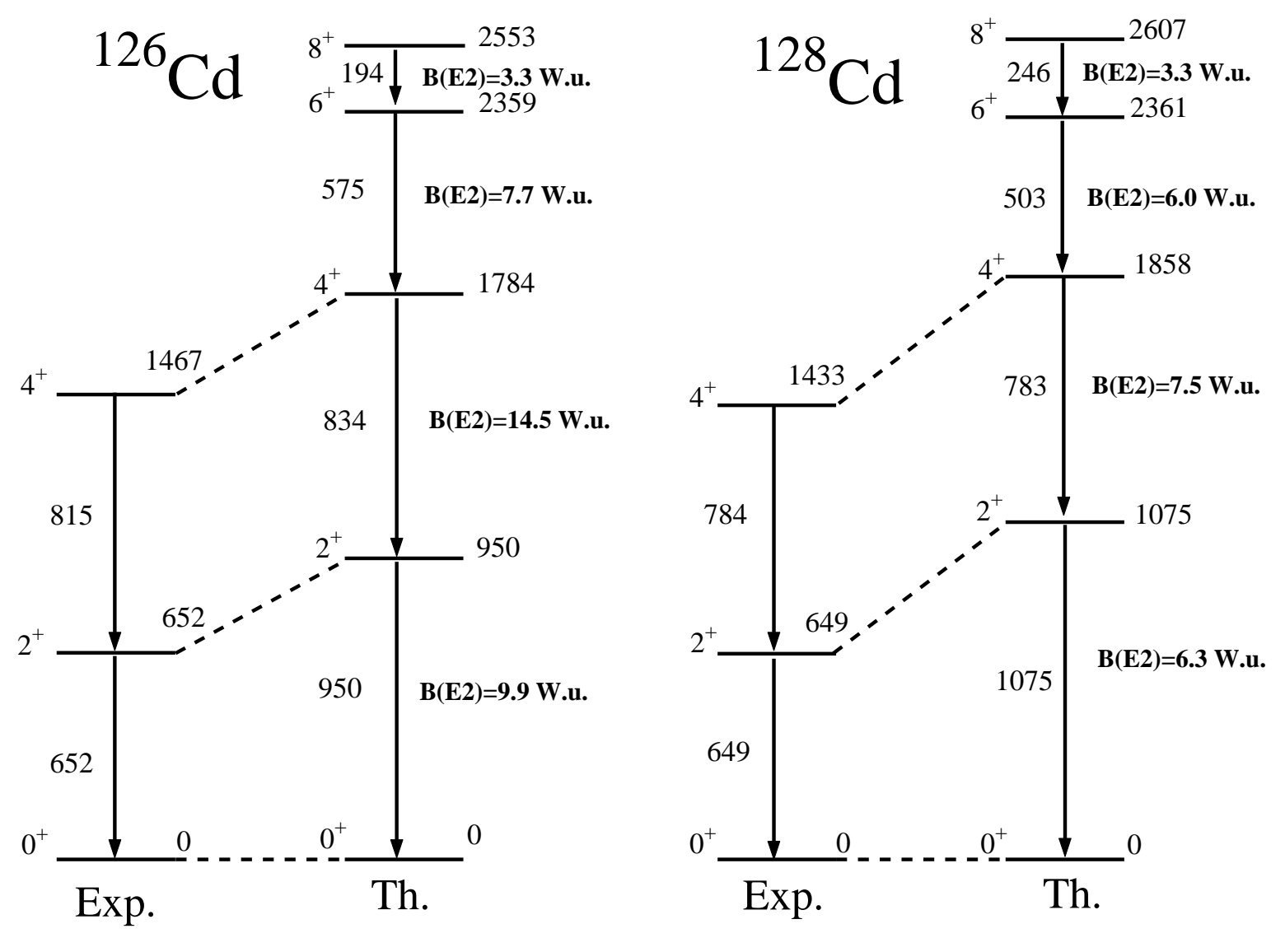

FIG. 7: Experimental and calculated energies for ${ }^{126,128} \mathrm{Cd}$.

ever, the present information on this nucleus is too scarce to conclude that the collectivity is a consequence of the shell structure and more experimental data are needed to clarify the situation.

\section{M2 AND E3 TRANSITION PROBABILITIES IN SN AND IN ISOTOPES}

In table III, all the available $M 2$ and $E 3$ transitions in heavy In and Sn isotopes are reported. Among these transitions, only the first one corresponds to a spin variation $\Delta \mathrm{I}=3$, while all the others have $\Delta \mathrm{I}=2$ and $M 2 / E 3$ admixtures are possible.

All these transitions take place between states dominated by configurations which differ by the replacement of an $h_{11 / 2}$ neutron with a $d_{3 / 2}$ one, or vice versa. As $M 2$ or E3 transitions are not possible between these configurations, admixtures with configurations involving the $g_{7 / 2}$ or $d_{5 / 2}$ orbits are necessary. These two orbits are far from the Fermi level in the Sn and In isotopes, and the admixtures are expected to be small. The experimental 

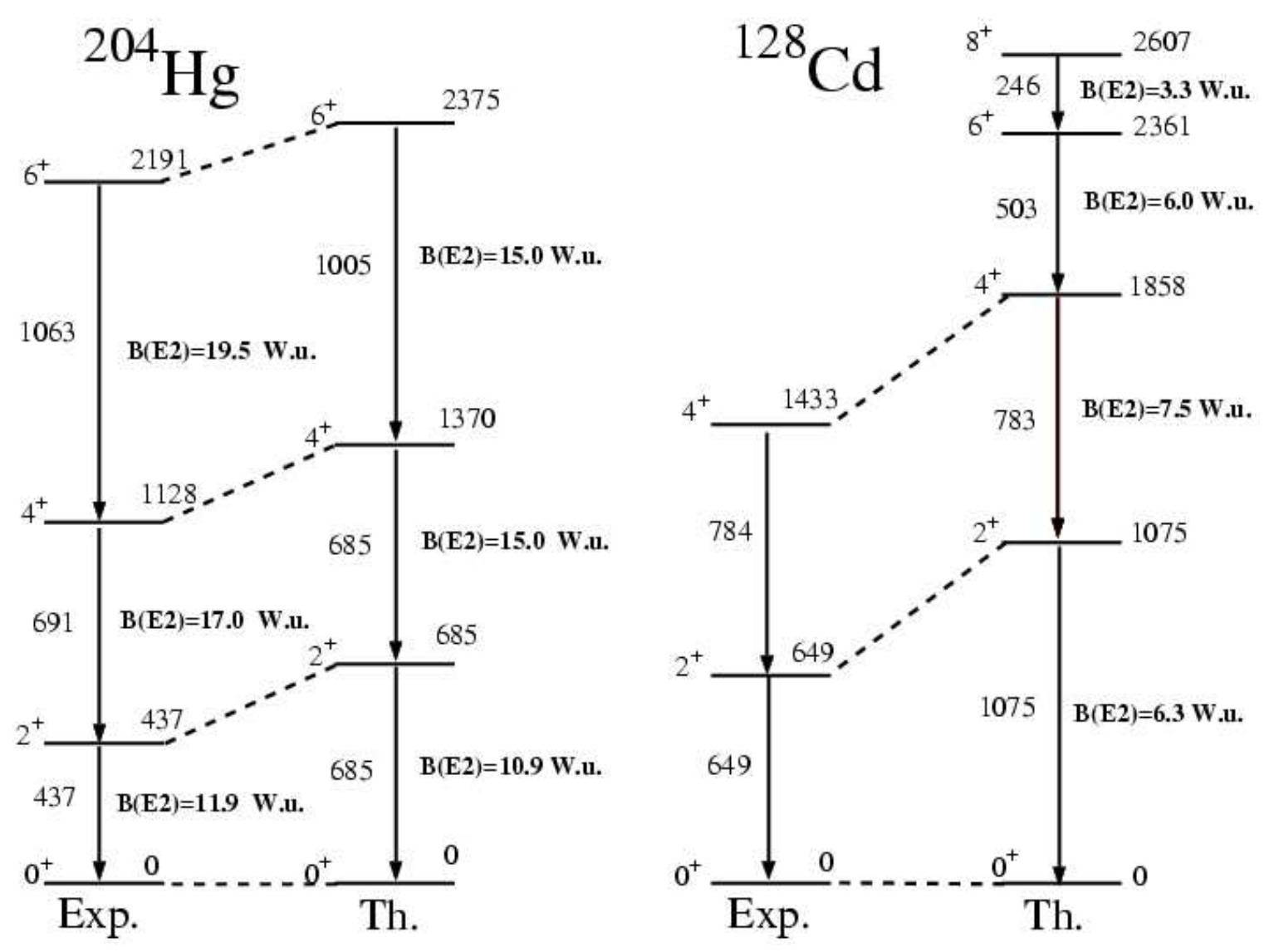

FIG. 8: Experimental and calculated energies for ${ }^{204} \mathrm{Hg}[20]$ and ${ }^{128} \mathrm{Cd}$.

$B(M 2)$ and $B(E 3)$ values reported in table III are obtained assuming no admixture of the multipolarities. The very small value $B(E 3)=0.06 \mathrm{~W}$.u. for the $29 / 2^{+} \rightarrow 23 / 2^{-}$transition in ${ }^{129}$ In gives the order of magnitude of the E3 strength in this region. Consequently, the very large values given in Table III for the other $B(E 3)$ transition probabilities in the In isotopes are not realistic, and one may conclude that the transitions have mainly an M2 character. By contrast, in the Sn isotopes it is not possible to exclude an E3 component in the transitions, and the $M 2$ strengths may be smaller than the reported values. It is interesting to note that the $B(M 2)$ values are between 10 and 100 times smaller in the Sn isotopes than in the In ones. This effect is likely to be related to the magic character of the Sn isotopes. The results of our shell-model calculations for the $B\left(M 2 ; 17 / 2^{-} \rightarrow 13 / 2^{+}\right)$and $B\left(E 3 ; 29 / 2^{+} \rightarrow 23 / 2^{-}\right)$transitions in ${ }^{129}$ In have been reported in Ref. [11] and are in very good agreement with experiment. As regards the other $B(E 3)$ transitions, our calculations predict very small values, typically $10^{-2}$ W.u. for the In isotopes and $10^{-1} \mathrm{~W}$. u. for the Sn isotopes. 
TABLE III: $B(M 2)$ and $B(E 3)$ values of $M 2 / E 3$ transitions in $125,126,128,129,130 \mathrm{In}$ and ${ }^{123,125,127,129} \mathrm{Sn}$. The values reported for ${ }^{129} \mathrm{In}$ are taken from [11]; the values reported for ${ }^{123,125,127,129} \mathrm{Sn}$ are taken from $[6,21]$.

\begin{tabular}{|c|c|c|c|c|}
\hline Nucleus & Energy (keV) & Transition & $B(M 2)$ W.u. & $B(E 3)$ W.u. \\
\hline${ }^{129} \mathrm{In}$ & 281 & $29 / 2^{+} \rightarrow 23 / 2^{-}$ & & 0.06 \\
\hline${ }^{129} \mathrm{In}$ & 333.8 & $17 / 2^{-} \rightarrow 13 / 2^{+}$ & 0.033 & 283 \\
\hline${ }^{130} \mathrm{In}$ & 388.8 & $3^{+} \rightarrow 1^{-}$ & 0.047 & 27.6 \\
\hline${ }^{128} \operatorname{In}$ & 247.9 & $1^{-} \rightarrow 3^{+}$ & 0.047 & 73 \\
\hline${ }^{126} \mathrm{In}$ & 243.3 & $1^{-} \rightarrow 3^{+}$ & 0.053 & 89 \\
\hline${ }^{125} \operatorname{In}$ & 151 & $25 / 2^{+} \rightarrow 21 / 2^{-}$ & 0.0015 & 43 \\
\hline${ }^{129} \mathrm{Sn}$ & 590 & $19 / 2^{+} \rightarrow 15 / 2^{-}$ & $<1.7 \times 10^{-4}$ & 0.49 \\
\hline${ }^{127} \mathrm{Sn}$ & 723 & $19 / 2^{+} \rightarrow 15 / 2^{-}$ & $1.6 \times 10^{-4}$ & 0.30 \\
\hline${ }^{125} \mathrm{Sn}$ & 806 & $19 / 2^{+} \rightarrow 15 / 2^{-}$ & $2.9 \times 10^{-4}$ & 0.47 \\
\hline${ }^{123} \mathrm{Sn}$ & 838 & $19 / 2^{+} \rightarrow 15 / 2^{-}$ & $1.8 \times 10^{-4}$ & 0.29 \\
\hline
\end{tabular}

\section{CONCLUSIONS}

The present delayed $\gamma$-rays and conversion electrons measurements of fission products have allowed us to complete the level schemes of the In isotopes in the mass range $A=123$ to 130. A new half-life was measured for ${ }^{123} \mathrm{In}$ and several half-lives known with large uncertainties were remeasured. We have found out that no $\mu$ s isomers exist in ${ }^{126,128} \mathrm{Cd}$ with half-lives longer than $0.5 \mu \mathrm{s}$. It was also shown that the $M 2$ transitions are much faster in the In isotopes than in the proton-magic Sn isotopes.

Along with the experimental work, we have performed realistic shell-model calculations for the odd-mass ${ }^{127} \mathrm{In}$, the even-mass ${ }^{126,128,130} \mathrm{In}$, and ${ }^{126,128} \mathrm{Cd}$. These calculations provide a satisfactory interpretation of ${ }^{129,130} \mathrm{In}$, but some discrepancies occur for the lighter isotopes. The calculations for ${ }^{126,128} \mathrm{Cd}$ predict short half-lives $(\sim 10 \mathrm{~ns})$ for the $8^{+}$states, which could explain why they have not been observed in the present work. We have also shown that these nuclei present some similarities with ${ }^{204} \mathrm{Hg}$. Both ${ }^{128} \mathrm{Cd}$ and ${ }^{204} \mathrm{Hg}$ have only two proton and two neutron holes inside doubly-magic cores and present a collective behavior. However, more experimental data, in particular $B(E 2)$ transition probabilities are necessary to test 
this hypothesis.

\section{Acknowledgments}

One of the authors (A. S.) acknowledges support from BMBF under grant O6K-167. We would like to thank Professor V. Rubchenya for providing calculated fission yields in the symmetry region. Professor M. Asghar is gratefully acknowledged for stimulating discussion and careful reading of the manuscript.

[1] J. Genevey and J. A. Pinston, J. Phys. G 30, R57 (2004).

[2] T. Kautzsch, W. B. Walters, M. Hannawald, K.-L. Kratz, V. I. Mishin, V. N. Fedoseyev, W. Böhmer, Y. Jading, P. Van Duppen, B. Pfeiffer, et al., Eur. Phys. J. A 9, 201 (2000).

[3] P. Hoff, B. Ekström, and B. Fogelberg, Nucl. Phys. A 459, 35 (1986).

[4] H. Huck, A. Jech, G. Martí, M. L. Pérez, J. J. Rossi, and H. M. Sofía, Phys Rev. C 39, 997 (1989).

[5] B. Fogelberg, H. Mach, H. Gausemel, J. P.Omtved, and K. A. Mezilev, in Nuclear Fission and Fission-Product Spectroscopy, edited by G. Fioni, H. Faust, S. Obersted, and F. Hambsch (American Institute of Physics, 1998), AIP Conference Proceedings 447, p. 191.

[6] H. Gausemel, B. Fogelberg, T. Engeland, M. Hjorth-Jensen, P. Hoff, H. Mach, K. A. Mezilev, and J. P. Omtvedt, Phys. Rev. C 69, 054307 (2004).

[7] M. Hellström, M. N. Mineva, A. Blazhev, H. J. Boardman, J. Ekman, K. Gladnishki, H. Grawe, J. Gerl, R. Page, Z. Podolyak, et al., in Proc. Third Int. Conf. Fission and Properties of the Neutron-Rich Nuclei, edited by J. Hamilton, A. Ramayya, and H. Carter (World Scientific, 2003), p. 22.

[8] M. Hellström, M. N. Mineva, A. Blazhev, H. J. Boardman, J. Ekman, J. Gerl, K. Glandishki, H. Grawe, R. Page, Z. Podolyak, et al., in Nuclear structure and dynamics at the limits: proceedings of the International Workshop XXXI on Gross Properties of Nuclei and Nuclear Excitations: Hirschegg, Austria, January 12-18, 2003, edited by H. Feldmeier (Imprint Darmstadt : Gesellschaft fr Schwerionenforsching (GSI), 2003), p. 72.

[9] R. Machleidt, Phys. Rev. C 63, 024001 (2001). 
[10] L. Coraggio, A. Covello, A. Gargano, N. Itaco, and T. T. S. Kuo, Phys. Rev. C 66, 064311 (2002).

[11] J. Genevey, J. A. Pinston, H. R. Faust, R. Orlandi, A. Scherillo, G. S. Simpson, I. S. Tsekhanovich, A. Covello, A. Gargano, and W. Urban, Phys. Rev. C 67, 054312 (2003).

[12] J. Genevey, J. A. Pinston, H. Faust, C. Foin, S. Obersted, and M. Rejmund, Eur. Phys. J. A 9, $191(2000)$.

[13] J. Eberth, G. Pascovici, H. G. Thomas, N. Warr, D. Weißhaar, D. Habs, P. Reiter, P. Thirolf, D. Schwalm, C. Gund, et al., Prog. Part. Nucl. Phys 46, 389 (2001).

[14] R. H. Mayer, D. T. Nisius, I. G. Bearden, P. Bhattacharyya, L. Ritchter, M. Sferrazza, Z. W. Grabowski, P. J. Daly, B. Fornal, I. Ahmad, et al., Phys. Lett. B 336, 308 (1994).

[15] B.Fogelberg, in Proc. Intern. Conf. Nuclear Data for Science and Technology, Mito, Japan (1988), p. 837.

[16] L. Coraggio, A. Covello, A. Gargano, N. Itaco, and T. T. S. Kuo, J. Phys. G 26, 1697 (2000).

[17] B. A. Brown, A. Etchegoyen, and W. D. M. Rae, MSU-NSCL (Report No. 524).

[18] I. Dillmann, K. L. Kratz, A. Wøhr, O. Arndt, B. A. Brown, P. Hoff, M. Hjorth-Jensen, U. Köster, A. N. Ostrowski, B. Pfeiffer, et al., Phys. Rev. Lett. 91, 162503 (2003).

[19] L. Spanier, K. Aleklett, B. Ekstrom, and B. Fogelberg, Nucl. Phys. A474, 359 (1987).

[20] L. Rydstrom, J. Blomqvist, R. J. Liotta, and C. Pomar, Nucl.Phys. A512, 217 (1990).

[21] J. A. Pinston, C. Foin, J. Genevey, R. Béraud, E. Chabanat, H. Faust, S. Oberstedt, and B. Weiss, Phys. Rev. C 61, 024312 (2000). 